\title{
Incidence and Risk of Atrial Fibrillation in Sleep-Disordered Breathing Without Coexistent Systemic Disease
} - Nationwide Longitudinal Cohort Study -

Tze-Fan Chao, MD; Chia-Jen Liu, MD; Su-Jung Chen, MD; Kang-Ling Wang, MD; Yenn-Jiang Lin, MD; Shih-Lin Chang, MD; Li-Wei Lo, MD; Yu-Feng Hu, MD; Ta-Chuan Tuan, MD; Tzeng-Ji Chen, MD; Chuen-Wang Chiou, MD; Shih-Ann Chen, MD

\begin{abstract}
Background: Although the link between sleep-disordered breathing (SDB) and atrial fibrillation (AF) has been reported, a population-based longitudinal cohort study was lacking. The goal of the present study was to investigate the AF risk carried by SDB, using the National Health Insurance Research Database in Taiwan.

Methods and Results: From 2000 to 2001, a total of 579,521 patients who had no history of cardiac arrhythmias or significant comorbidities were identified. Among them, 4,082 subjects with the diagnosis of SDB were selected as the study group, and the remaining 575,439 subjects constituted the control group. The study endpoint was the occurrence of new-onset AF. During a follow-up of $9.2 \pm 2.0$ years, there were 4,023 patients $(0.7 \%)$ experiencing new-onset AF. The occurrence rate of AF was higher in patients with SDB compared to those without it $1.3 \% \mathrm{vs}$. $0.7 \%, P<0.001$ ). The AF incidences were 1.38 and 0.76 per 1,000 person-years for patients with and without SDB, respectively. After an adjustment for age and sex, SDB was a significant risk factor of AF with a hazard ratio of 1.536. The AF risk increased with increasing clinical severity of SDB, represented by the requirement of continuous positive airway pressure use.
\end{abstract}

Conclusions: SDB itself, without the coexistence of other systemic diseases, was a risk factor of AF. (Circ J 2014; 78: 2182-2187)

Key Words: Atrial fibrillation; Incidence; Sleep-disordered breathing

A trial fibrillation $(\mathrm{AF})$ is the most common sustained cardiac arrhythmia, and is associated with marked morbidity, mortality, and socioeconomic burden. ${ }^{1,2}$ The age-adjusted incidence of AF significantly increased from 3.04 to 3.68 per 1,000 person-years in the USA. ${ }^{3}$ The incidence of $\mathrm{AF}$ is lower in Asia. ${ }^{4}$ In a nationwide cohort of 702,502 participants in Taiwan, the AF incidence was around 1.5 per 1,000 person-years, and was much lower in patients with a CHADS2 (Congestive Heart Failure, Hypertension, Age, Diabetes, Stroke/ Transient Ischemic Attack) score of zero ( 0.77 per 1,000 person-years). ${ }^{5}$ Traditional risk factors for $\mathrm{AF}$ include advanced age, male gender, hypertension, diabetes mellitus, heart failure and end-stage renal disease. ${ }^{5-7}$ Recently, some novel predis- posing factors for $\mathrm{AF}$ were identified, such as obesity, metabolic syndrome and use of steroids or non-steroidal anti-inflammatory drugs. ${ }^{8-10}$

Sleep-disordered breathing (SDB) has been reported to be associated with various cardiovascular diseases, including hypertension, coronary artery disease, heart failure, ventricular arrhythmias, stroke and sudden cardiac arrest. ${ }^{11-14}$ In recent years, there has been increasing awareness of the possible link between SDB and AF. ${ }^{15-18}$ A previous study, however, showed that SDB was not a risk factor of $\mathrm{AF}$ in patients without evident cardiovascular diseases, ${ }^{16}$ raising the question of whether SDB alone is a predisposing factor for AF. Also, the temporal relationship between SDB and AF has not been studied in a population-

Received February 25, 2014; revised manuscript received June 7, 2014; accepted June 9, 2014; released online July 23, 2014 Time for primary review: 2 days

Division of Cardiology (T.-F.C., K.-L.W., Y.-J.L., S.-L.C., L.-W.L., Y.-F.H., T.-C.T., S.-A.C.), Division of Hematology and Oncology (C.-J.L.), Division of Infectious Diseases (S.-J.C.), Department of Medicine, Taipei Veterans General Hospital, Taipei; Department of Family Medicine, Taipei Veterans General Hospital, Taipei (T.-J.C.); Institute of Clinical Medicine, and Cardiovascular Research Center, National Yang-Ming University, Taipei (T.-F.C., K.-L.W., Y.-J.L., S.-L.C., L.-W.L., Y.-F.H., T.-C.T., C.-W.C., S.-A.C.); Division of Cardiology, Taipei Municipal Gan-Dau Hospital, Taipei (T.-F.C., T.-C.T.); and Division of Cardiology, Kaohsiung Veterans General Hospital, Kaohsiung (C.-W.C.), Taiwan

Mailing address: Shih-Ann Chen, MD, Division of Cardiology, Department of Medicine, Taipei Veterans General Hospital, No. 201, Sec. 2, Shih-Pai Road, Taipei, Taiwan. E-mail: epsachen@ms41.hinet.net and Chuen-Wang Chiou, MD, Division of Cardiology, Kaohsiung Veterans General Hospital, 386, Ta-Chung 1 ${ }^{\text {st }}$ Road, Kaohsiung, Taiwan. E-mail: cwchiou@isca.vghks.gov.tw

ISSN-1346-9843 doi:10.1253/circj.CJ-14-0222

All rights are reserved to the Japanese Circulation Society. For permissions, please e-mail: cj@j-circ.or.jp 


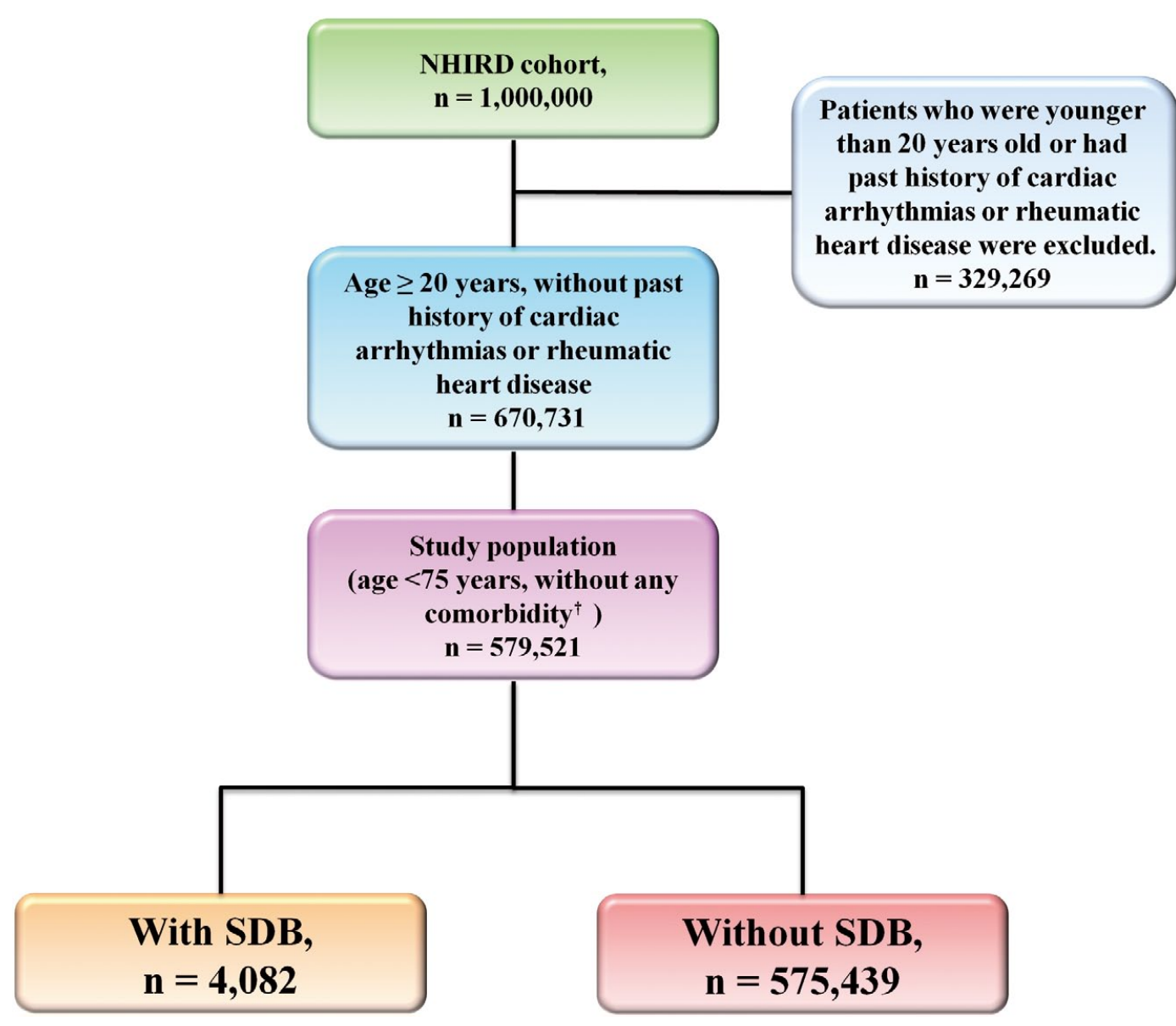

Figure 1. Subject enrollment. A total of 579,521 patients younger than 75 years old without any significant comorbidity were enrolled in the present study. Among the study population, 4,082 patients had the diagnosis of sleep-disordered breathing. ${ }^{+}$Comorbidities include hypertension, diabetes mellitus, congestive heart failure, ischemic stroke or transient ischemic attack, ischemic heart disease, chronic kidney disease, chronic pulmonary disease (asthma or chronic obstructive pulmonary disease), peripheral arterial disease, malignancy and systemic autoimmune diseases. NHIRD, National Health Insurance Research Database; SDB, sleep-disordered breathing.

based longitudinal cohort, which would have less selection bias and could more accurately represent the real-world condition. Therefore, the goal of the present study was to investigate the risk of AF carried by SDB in a nationwide database, and to determine whether SDB is a novel risk factor for AF in a population without significant comorbidities.

\section{Methods}

\section{Database}

This study used the National Health Insurance Research Database (NHIRD) released by the Taiwan National Health Research Institutes (NHRI). The National Health Insurance (NHI) system is a mandatory universal health insurance program that offers comprehensive medical care coverage to all Taiwanese residents. The NHIRD is a cohort dataset that contains all the medical claims data for 1,000,000 beneficiaries, who were randomly sampled from the 25.68 million enrollees under the NHI program. These random samples have been confirmed by the NHRI to be representative of the Taiwanese population. In this cohort dataset, the patients' original identi- fication numbers have been encrypted to protect their privacy, but the encrypting procedure was consistent, so that a linkage of the claims belonging to the same patient is feasible within the NHI database and can be followed continuously. The database, with its large sample size provided an excellent opportunity to study the relationship between SDB and AF.

\section{Subjects}

From 1 January 2000 to 31 December 2001, 670,731 patients who were aged $\geq 20$ years and had no past history of cardiac arrhythmias or rheumatic heart disease were identified from the NHIRD. A total of 579,521 patients who were younger than 75 years old and had no significant comorbidities, including hypertension, diabetes mellitus, congestive heart failure, ischemic stroke or transient ischemic attack, ischemic heart disease, chronic kidney disease, chronic pulmonary disease (asthma or chronic obstructive pulmonary disease), peripheral arterial disease, malignancy and systemic autoimmune diseases, were selected. From them, 4,082 subjects who had the diagnosis of SDB were enrolled as the study group, and the remaining 575,439 subjects constituted the control group. SDB was diagnosed using 


\begin{tabular}{|lccc|}
\hline \multicolumn{2}{|c|}{ Table 1. Age and Gender vs. Presence of SDB } & & \\
\multicolumn{1}{|c|}{ Variables } & $\begin{array}{c}\text { Study group (with SDB) } \\
(\mathbf{n}=\mathbf{4 , 0 8 2})\end{array}$ & $\begin{array}{c}\text { Control group (without SDB) } \\
(\mathbf{n = 5 7 5 , 4 3 9 )}\end{array}$ & P-value \\
Mean age (years) & $43.6 \pm 12.6$ & $38.9 \pm 13.1$ & $<0.001$ \\
Age distribution (years) & & & $<0.01$ \\
$20-40$ & $1,610(39.4)$ & $324,163(56.3)$ & \\
$40-60$ & $1,959(48.0)$ & $199,145(34.6)$ & \\
$60-74$ & $513(12.6)$ & $52,131(9.1)$ & \\
Male & $2,604(63.8)$ & $294,458(51.2)$ & $<0.001$ \\
\hline
\end{tabular}

Data given as mean \pm SD or $n(\%)$. SDB, sleep-disordered breathing.

\begin{tabular}{|c|c|c|c|}
\hline \multirow{2}{*}{ Variable } & \multirow{2}{*}{ All $(n=579,521)$} & \multicolumn{2}{|c|}{ SDB } \\
\hline & & Yes $(n=4,082)$ & No $(n=575,439)$ \\
\hline \multicolumn{4}{|l|}{ AF } \\
\hline Yes & $4,023(0.7)$ & $53(1.3)^{\dagger}$ & $3970(0.7)$ \\
\hline No & $575,498(99.3)$ & $4,029(98.7)$ & $571,469(99.3)$ \\
\hline Crude HR $(95 \% \mathrm{Cl})$ & & \multicolumn{2}{|c|}{1.815 (1.384-2.380); $P<0.001$} \\
\hline Adjusted HR $(95 \% \mathrm{Cl})^{\ddagger}$ & & \multicolumn{2}{|c|}{1.536 (1.171-2.014); $P=0.002$} \\
\hline
\end{tabular}

Data given as $n(\%)$. Mean follow-up was $9.2 \pm 2.0$ years. ${ }^{\mathrm{P}} \mathrm{P}<0.001$ in comparison with patients without $\mathrm{SDB}$. ${ }^{\ddagger}$ Adjustment for age and gender.

$\mathrm{AF}$, atrial fibrillation; $\mathrm{Cl}$, confidence interval; $\mathrm{HR}$, hazard ratio. Other abbreviation as in Table 1.

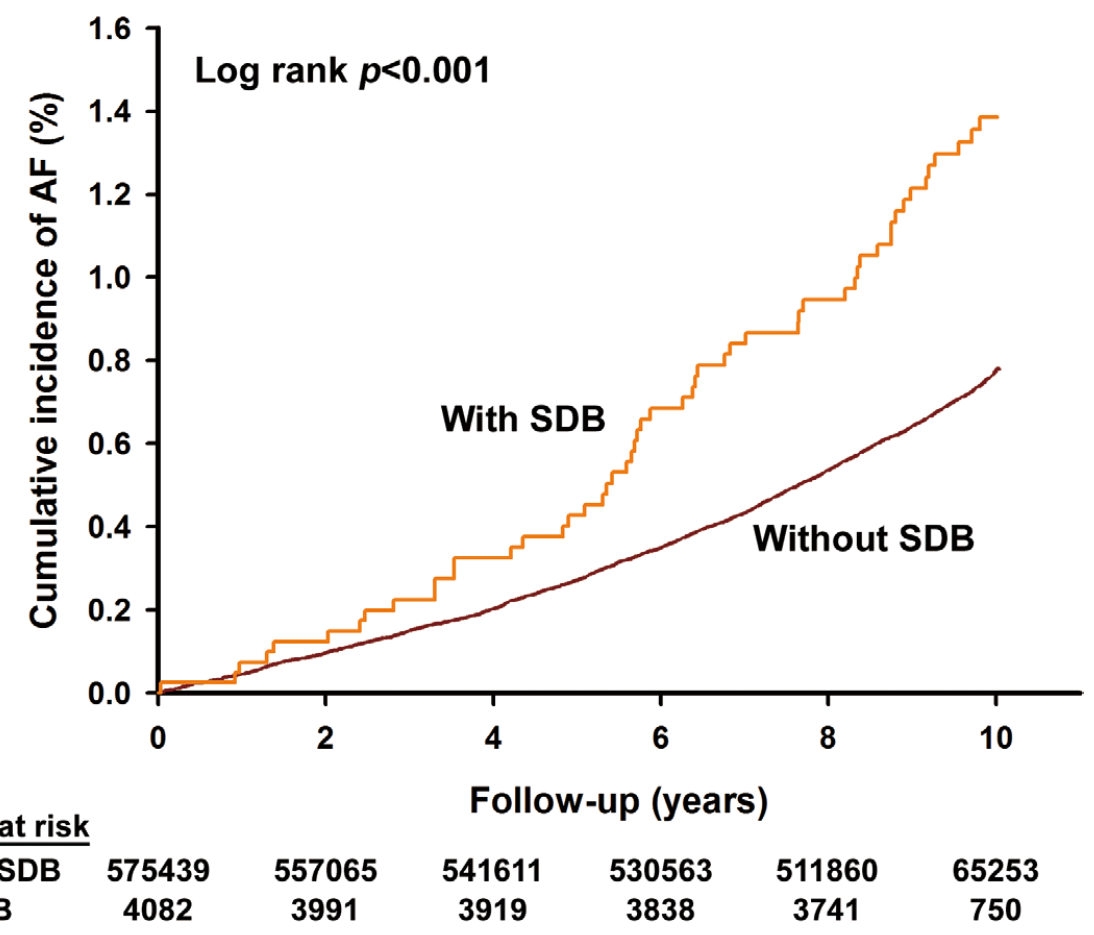

Figure 2. Cumulative frequency curve for new-onset atrial fibrillation (AF). Patients with sleep-disordered breathing (SDB) had a higher rate of new-onset AF during the follow-up period.

the International Classification of Diseases, Ninth Revision, Clinical Modification (ICD-9-CM) codes (780.51, 780.53, and 780.57), which theoretically represent an apnea-hypopnea index $>5 .{ }^{19-22}$ A flowchart of the enrollment of study patients is shown in Figure 1.
The study endpoint was the occurrence of new-onset AF (ICD-9-CM code 427.31) during the follow-up period. To ensure accuracy of diagnosis, we defined patients as having AF only when it was a discharge diagnosis or confirmed more than twice in the outpatient department. ${ }^{23}$ The diagnostic ac- 


\begin{tabular}{lcc} 
Table 3. Incidence of AF vs. Presence of SDB & \multicolumn{2}{c|}{ Groups } \\
\cline { 2 - 3 } & \multicolumn{3}{c}{ Study group (with SDB) } & Control group (without SDB) \\
No. new-onset AF & 53 & 3,970 \\
No. patients & 4,082 & 575,439 \\
Person-years & 38,371 & $5,236,495$ \\
Incidence $^{\dagger}$ & 1.38 & 0.76 \\
\hline
\end{tabular}

${ }^{\dagger} \mathrm{AF}$ cases per 1,000 person-years of follow-up. Abbreviation as in Tables 1,2.

\begin{tabular}{|c|c|c|c|}
\hline Groups & $\mathrm{HR}^{\dagger}$ & $95 \% \mathrm{Cl}$ & P-value \\
\hline Without SDB (reference group) & 1 & - & - \\
\hline SDB, CPAP not indicated & 1.457 & $1.099-1.932$ & 0.009 \\
\hline SDB, CPAP indicated & 4.507 & $1.691-12.010$ & 0.003 \\
\hline
\end{tabular}

${ }^{\dagger}$ Adjusted for age and gender.

CPAP, continuous positive airway pressure. Other abbreviation as in Tables 1,2.

curacy of AF using this definition in NHIRD has been validated previously. ${ }^{24,25}$ In the Chang et al study, which reviewed the medical records of 660 patients who fitted the diagnosis of AF defined by ICD-9-CM codes, the diagnostic accuracy of AF was around $90 \% .^{25}$

Continuous positive airway pressure (CPAP) applied a constant level of positive pressure at the airway opening during spontaneous breathing, and represented a treatment indicated for patients with SDB who have not responded to conservative therapies, such as behavior modification and oral appliances. In order to understand the relationship between the clinical severity of SDB and the risk of $\mathrm{AF}$, we analyzed whether CPAP was used for patients with SDB, and regarded it as a proxy of disease severity.

\section{Statistical Analysis}

The data are presented as mean \pm SD for normally distributed continuous variables and as proportions for categorical variables. The differences between continuous values were assessed using an unpaired 2-tailed t-test. The chi-squared test was used for comparisons of nominal variables. The AF-event curve was plotted and the statistical significance was examined using logrank test. The risk of patients with or without SDB in developing AF is expressed as the hazard ratio (HR), which was analyzed using Cox regression analysis. Statistical significance was set at $\mathrm{P}<0.05$ and all statistical analysis was carried out using SPSS 17.0 (SPSS, Chicago, IL, USA).

\section{Results}

The mean subject age was 38.9 \pm 13.1 years (range, $20-74$ years) and men accounted for $51.3 \%$ of the patients. Data on age and gender are listed in Table 1. Patients with SDB were older than those without it, and male gender was more prevalent among the study group.

During the period from January 2001 to December 2009 (mean follow-up, 9.2 \pm 2.0 years), there were 4,023 patients $(0.7 \%)$ experiencing new-onset AF. The occurrence rate of AF was higher in patients with SDB compared to those without it ( $1.3 \%$ vs. $0.7 \%, \mathrm{P}<0.001$; Table 2 ). The cumulative frequency curve of incident AF for subjects with and without SDB is shown in Figure 2. The incidence of AF was around 1.38 and 0.76 per 1,000 person-years for patients with and without SDB, respectively (Table 3). Table 2 also lists the crude and adjusted HR for the development of AF of these 2 groups. After adjustment for age and sex, SDB was a significant risk factor of new-onset AF with a HR of 1.536 (95\% confidence interval [95\% CI]: 1.171-2.014, $\mathrm{P}=0.002$ ) on multivariate Cox regression analysis.

In the subgroup analysis of patients with SDB stratified by whether CPAP use was indicated, the adjusted risk of AF was significantly higher in patients who needed CPAP treatment compared to the control group (without SDB) with a HR of 4.507 (95\% CI: 1.691-12.010, $\mathrm{P}=0.003$; Table 4). For patients with SDB who did not require CPAP use, the risk of AF was still higher than those without SDB (HR, 1.457; 95\% CI: 1.099$1.932, \mathrm{P}=0.009)$.

\section{Discussion}

\section{Main Findings}

In this large-scale nationwide population-based cohort study, we investigated the association of SDB and AF among healthy subjects who were younger than 75 years old and who had no significant comorbidities. The main findings were as follows: (1) the incidence of AF was around 1.38 per 1,000 person-years for patients with SDB; (2) SDB significantly predisposed patients to develop AF, and was a novel risk factor of new-onset AF; and (3) AF risk increased with increasing clinical severity of SDB, represented by the requirement of CPAP use.

\section{SDB and Risk of AF}

The present study has shown that AF incidence was significantly higher in patients with SDB than control subjects (1.38 vs. 0.76 per 1,000 person-years). To the best of our knowledge, this is the first study reporting the incidence of AF in patients with SDB alone in a population-based cohort. But what are the possible mechanisms of the link between SDB and AF? In the Dimitri et al study, which enrolled a total of 40 paroxysmal AF patients receiving catheter ablation (20 subjects had SDB), biatrial electroanatomic mapping and electrophysiological study showed that SDB was associated with significant atrial remodeling, characterized by atrial enlargement, voltage reduction, abnormal conductions, and longer sinus node recovery time. ${ }^{26}$ In a recent animal study from Stanley Nattel's group, intubated rats underwent hemodynamic and echocardiographic studies 
after obstructive sleep apnea was mimicked by stopping the ventilator and closing the airway for $40 \mathrm{~s}^{27}$ The results suggested that forced respiration-induced acute left atrial stretch superimposed on left ventricular diastolic dysfunction may be central to AF promotion in SDB. In addition to the left atrial remodeling, Ghias et al reported that SDB-induced AF may be attenuated by ablation of the pulmonary artery-associated ganglionated plexi or pharmacological inhibition of autonomic inputs in an animal model similar to SDB, suggesting that an autonomic link may exist between SDB and AF. ${ }^{28,29}$ Further, the inflammatory responses caused by SDB, which were associated with atrial remodeling, ${ }^{30}$ arousal responses, the fluctuation of heart rate and hypoxic stress, may also predispose patients with SDB to AF. Taken together, structural and electrical remodeling of the LA due to an increased inflammatory status and repeated atrial stretch and autonomic activation during the apnea episodes may play an important role in the pathogenesis of SDB-related AF.

\section{Previous Studies}

In addition to the traditional factors associated with AF, such as hypertension, diabetes and advanced age, many investigators devoted themselves to finding more novel risk factors. Through the identification of more and more predisposing factors to $\mathrm{AF}$, physicians have been able to identify patients at risk of AF more accurately. The present results show that SDB itself is an important risk factor of AF, a finding further supported by the observation that the risk of $\mathrm{AF}$ is even higher in patients with SDB with a high clinical severity.

Although the close association between SDB and AF has been discussed and become well-accepted in recent years, we are surprised to find that there have been only several studies investigating whether SDB is a risk factor of AF in the literature, which are summarized in Table S1. It should be noted that most of these studies were cross-sectional observations, ${ }^{15,16,18}$ and may not be able to indicate a temporal relationship between SDB and AF. The Gami et al study, which enrolled a total of 3,542 adults referred for a diagnostic polysomnogram from 1987 to 2003 at the Mayo Clinic, represented the only one longitudinal cohort study investigating this issue. ${ }^{17} \mathrm{SDB}$, however, was present in 2,626 subjects (74\%) in the Gami et al study, implying that important selection biases could be present and the results may not be generalized to real-world clinical practice. In the present study, we explored the temporal relationship between SDB and AF in a nationwide population-based cohort, which may minimize the risk of significant selection biases and facilitate understanding of the SDB-related incidence of AF. In contrast to the Porthan et al study showing that SDB was not significantly associated with lone $A F,{ }^{16}$ the present study indicates that SDB is an important predisposing factor to $\mathrm{AF}$ among patients without significant comorbidities, with a HR of 1.536. The negative correlation between SDB and AF in the Porthan et al study may be due to the small sample size, with only $59 \mathrm{AF}$ and 56 non-AF patients, in contrast to the present study, which had a large patient number.

\section{Clinical Applications}

The current study has shown that SDB itself can cause AF without the coexistence of other systemic diseases. This finding has not been clearly mentioned before, and provides convincing evidence to support the concept that SDB is a novel risk factor of AF. For patients with SDB who have had ischemic stroke, aggressive examinations should be considered to detect $\mathrm{AF}$ even in the absence of any systemic disease. SDB should also be regarded as a potential cause of $\mathrm{AF}$ and be routinely screened for when managing AF patients, especially those without any comorbidity and younger than 75 years old.

\section{Study Limitations}

There were several limitations of the present study. First, the occurrence of AF was based on the diagnostic code registered by the treating physicians, and was not further checked externally. This, however, is a common limitation, which was also noted in a previous study, ${ }^{31}$ and the diagnosis of AF in the present investigation was repeatedly confirmed more than twice to minimize diagnostic bias. Second, information on type of SDB (obstructive or central type) was not available in this nationwide registry. Nonetheless, more than $90 \%$ of patients with SDB referred for polysomnography had the obstructive type, ${ }^{32}$ and central sleep apnea was also reported to be associated with AF. ${ }^{33}$ Therefore, the type of SDB may not be an important factor that could confound or change the present results. The relationship between AF and central sleep apnea, however, could be bidirectional, with each leading to the other, and further study is necessary to determine whether obstructive or central SDB differs in causing AF. Third, patients enrolled in the present study without significant comorbidity at baseline could have developed new-onset cardiovascular diseases during the subsequent follow-up. Therefore, we were not able to ascertain that the occurrence of AF was solely attributed to SDB. Cardiovascular disease, however, would theoretically develop in both the study and control groups, and may not significantly confound the analysis comparing the risk of AF between patients with or without SDB. Fourth, the diagnosis of SDB was based on the same ICD-9-CM codes as that used in previous studies from Taiwan, ${ }^{19-22}$ and the data on the devices used for diagnosis and the severity of SDB (such as apnea-hypopnea index) were not recorded in this registry database. The diagnostic accuracy of SDB in NHIRD, however, has been validated in a single-center cohort previously, and around $83 \%$ of the diagnosis was confirmed on overnight polysomnography, with approximately $90 \%$ of them having an apnea-hypopnea index $>5 .{ }^{22}$ Last, we analyzed data on CPAP use only when study subjects were enrolled; whether patients continued to receive CPAP treatment during the follow-up period was uncertain. Consequently, it is unclear whether patients with SDB who received regular CPAP treatment had a lower risk of AF compared to patients with a similar severity of SDB but without long-term CPAP use. Decision-making regarding CPAP was carried out by physicians without a uniform rule, and a selection bias may exist.

\section{Conclusions}

The incidence of AF was around 1.38 per 1,000 patient-years among subjects with SDB alone, which was much higher than in those without it (0.76 per 1,000 person-years). SDB itself, without the coexistence of other systemic diseases, is a novel risk factor of $\mathrm{AF}$, and the risk increased with increasing clinical severity of SDB. The important link between SDB and AF should always be kept in mind when managing SDB and AF patients.

\section{Acknowledgments}

This work was supported in part by grants from the National Science Council (NSC98-2410-H-010-003-MY2), and Taipei Veterans General Hospital (V99C1-140, V99A-153, and V100D-002-3, V101D-001-2, and V102B-025).

\section{References}

1. Wolf PA, Mitchell JB, Baker CS, Kannel WB, D'Agostino RB. Impact of atrial fibrillation on mortality, stroke, and medical costs. 
Arch Intern Med 1998; 158: 229-234.

2. Chugh SS, Blackshear JL, Shen WK, Hammill SC, Gersh BJ. Epidemiology and natural history of atrial fibrillation: Clinical implications. J Am Coll Cardiol 2001; 37: 371-378.

3. Miyasaka Y, Barnes ME, Gersh BJ, Cha SS, Bailey KR, Abhayaratna WP, et al. Secular trends in incidence of atrial fibrillation in Olmsted County, Minnesota, 1980 to 2000, and implications on the projections for future prevalence. Circulation 2006; 114: 119-125.

4. Chiang CE, Zhang S, Tse HF, Teo WS, Omar R, Sriratanasathavorn C. Atrial fibrillation management in Asia: From the Asian expert forum on atrial fibrillation. Int J Cardiol 2013; 164: 21-32.

5. Chao TF, Liu CJ, Chen SJ, Wang KL, Lin YJ, Chang SL, et al CHADS(2) score and risk of new-onset atrial fibrillation: A nationwide cohort study in Taiwan. Int J Cardiol 2013; 168: 1360-1363.

6. Lip GY, Tse HF, Lane DA. Atrial fibrillation. Lancet 2012; 379: 648-661.

7. Schnabel RB. Can we predict the occurrence of atrial fibrillation? Clin Cardiol 2012; 35 (Suppl 1): 5-9.

8. Watanabe H, Tanabe N, Watanabe T, Darbar D, Roden DM, Sasaki $\mathrm{S}$, et al. Metabolic syndrome and risk of development of atrial fibrillation: The Niigata preventive medicine study. Circulation 2008; 117: $1255-1260$.

9. De Caterina R, Ruigomez A, Rodriguez LA. Long-term use of antiinflammatory drugs and risk of atrial fibrillation. Arch Intern Med 2010; 170: $1450-1455$

10. Chao TF, Liu CJ, Chen SJ, Wang KL, Lin YJ, Chang SL, et al. The association between the use of non-steroidal anti-inflammatory drugs and atrial fibrillation: A nationwide case-control study. Int J Cardiol 2013; 168: $312-316$

11. Monahan K, Redline S. Role of obstructive sleep apnea in cardiovascular disease. Curr Opin Cardiol 2011; 26: 541 -547.

12. Sakakibara M, Yamada S, Kamiya K, Yokota T, Oba K, Tsutsui H. Sleep-disordered breathing is an independent risk factor of aborted sudden cardiac arrest in patients with coronary artery spasm. Circ $J$ 2012; 76: 2204-2210.

13. Oldenburg O. Cheyne-Stokes respiration in chronic heart failure: Treatment with adaptive servoventilation therapy. Circ J 2012; 76: $2305-2317$.

14. Yamada S, Suzuki H, Kamioka M, Suzuki S, Kamiyama Y, Yoshihisa A, et al. Sleep-disordered breathing increases risk for fatal ventricular arrhythmias in patients with chronic heart failure. Circ J 2013; 77: $1466-1473$.

15. Gami AS, Pressman G, Caples SM, Kanagala R, Gard JJ, Davison $\mathrm{DE}$, et al. Association of atrial fibrillation and obstructive sleep apnea. Circulation 2004; 110: $364-367$

16. Porthan KM, Melin JH, Kupila JT, Venho KK, Partinen MM. Prevalence of sleep apnea syndrome in lone atrial fibrillation: A casecontrol study. Chest 2004; 125: 879-885.

17. Gami AS, Hodge DO, Herges RM, Olson EJ, Nykodym J, Kara T, et al. Obstructive sleep apnea, obesity, and the risk of incident atrial fibrillation. J Am Coll Cardiol 2007; 49: 565-571.

18. Stevenson IH, Teichtahl H, Cunnington D, Ciavarella S, Gordon I, Kalman JM. Prevalence of sleep disordered breathing in paroxysmal and persistent atrial fibrillation patients with normal left ventricular function. Eur Heart J 2008; 29: 1662-1669.

19. Chou KT, Huang CC, Chen YM, Su KC, Shiao GM, Lee YC, et al.
Sleep apnea and risk of deep vein thrombosis: A non-randomized, pair-matched cohort study. Am J Med 2012; 125: 374-380.

20. Shiao TH, Liu CJ, Luo JC, Su KC, Chen YM, Chen TJ, et al. Sleep apnea and risk of peptic ulcer bleeding: A nationwide populationbased study. Am J Med 2013; 126: 249-255.

21. Huang CY, Chen YT, Wu LA, Liu CJ, Chang SC, Perng DW, et al. Sleep apnoea patients have higher mortality when confronting sepsis. Eur J Clin Invest 2014; 44: 38-45.

22. Su VY, Liu CJ, Wang HK, Wu LA, Chang SC, Perng DW, et al. Sleep apnea and risk of pneumonia: A nationwide population-based study. CMAJ 2014; 186: 415-421.

23. Chen SJ, Liu CJ, Chao TF, Wang KL, Chen TJ, Chou P, et al. Dental scaling and atrial fibrillation: A nationwide cohort study. Int J Cardiol 2013; 168: 2300-2303.

24. Lin LJ, Cheng MH, Lee CH, Wung DC, Cheng CL, Kao Yang YH. Compliance with antithrombotic prescribing guidelines for patients with atrial fibrillation: A nationwide descriptive study in Taiwan. Clin Ther 2008; 30: 1726-1736.

25. Chang CH, Lee YC, Tsai CT, Chang SN, Chung YH, Lin MS, et al. Continuation of statin therapy and a decreased risk of atrial fibrillation/ flutter in patients with and without chronic kidney disease. Atherosclerosis 2014; 232: 224-230.

26. Dimitri H, Ng M, Brooks AG, Kuklik P, Stiles MK, Lau DH, et al. Atrial remodeling in obstructive sleep apnea: Implications for atrial fibrillation. Heart Rhythm 2012; 9: 321-327.

27. Iwasaki YK, Shi Y, Benito B, Gillis MA, Mizuno K, Tardif JC, et al. Determinants of atrial fibrillation in an animal model of obesity and acute obstructive sleep apnea. Heart Rhythm 2012; 9: 1409-1416.

28. Ghias M, Scherlag BJ, Lu Z, Niu G, Moers A, Jackman WM, et al. The role of ganglionated plexi in apnea-related atrial fibrillation. $J$ Am Coll Cardiol 2009; 54: 2075-2083.

29. Asirvatham SJ, Kapa S. Sleep apnea and atrial fibrillation: The autonomic link. J Am Coll Cardiol 2009; 54: 2084-2086.

30. Burioka N, Koyanagi S, Fukuoka Y, Okazaki F, Fujioka T, Kusunose $\mathrm{N}$, et al. Influence of intermittent hypoxia on the signal transduction pathways to inflammatory response and circadian clock regulation. Life Sci 2009; 85: 372-378

31. Alonso A, Agarwal SK, Soliman EZ, Ambrose M, Chamberlain AM, Prineas RJ, et al. Incidence of atrial fibrillation in whites and AfricanAmericans: The Atherosclerosis Risk in Communities (ARIC) study. Am Heart J 2009; 158: 111-117.

32. Bixler EO, Vgontzas AN, Ten Have T, Tyson K, Kales A. Effects of age on sleep apnea in men: I. Prevalence and severity. Am J Respir Crit Care Med 1998; 157: 144-148.

33. Leung RS, Huber MA, Rogge T, Maimon N, Chiu KL, Bradley TD. Association between atrial fibrillation and central sleep apnea. Sleep 2005; 28: $1543-1546$.

\section{Supplementary Files}

Supplementary File 1

Table S1. Studies on SDB and AF

Please find supplementary file(s);

http://dx.doi.org/10.1253/circj.CJ-14-0222 